\title{
REARRANGEMENT OF LATTICE PARTICLES
}

\author{
M. MURASKIN \\ University of North Dakota \\ Physics Department \\ Grand Forks, ND 58201
}

(Received May 9, 1991 and in revised form October 3, 1991)

\begin{abstract}
We have studied origin point data which lead to soliton loop lattice systems when we specify an integration path in no integrability Aesthetic Field Theory. When we applied the integration scheme developed in previous paper we found that the solitons get rearranged. Close to the origin we saw a system more disorderly than the lattice. However, farther from the origin in two dimensional maps the location of planar maxima (minima) for fixed $y$ became regular. In this paper, we investigate various approaches with the aim of enlarging the nonsymmetric regions. Integrating in $z$ did not lead to an enlarged nonsymmetric region. We were able to enlarge the region by altering the magnitudes appearing in the origin point data. It is not clear if we can continually enlarge the nonsymmetric region by this method. We studied what we call an "imperfect" lattice which in a coarse sense can be thought of as being comprised of soliton loops when we specify an integration path. Here the integration scheme did not lead to an exact symmetry, but there was a repeat of "type" structures (as indicated by observations of contour lines in the maps). We then extended the system to higher dimensions. In particular, we studied a complex six dimensional space which is a natural extension of Minkowski space as an example. The system studied gave rise to a loop lattice, but with magnitudes of maxima (minima) of the different loops varying in an oscillatory way. When we applied the integration scheme to this system wo found no sign of the previously discussed symmetry in the domain studied although the system is not free from other regularities (this is also the case when magnitudes are altered).
\end{abstract}

KEY WORDS AND PHRASES. Numerical approximation to solutions of partial differential equations.

AMS SUBJECT CLASSIFICATION CODES. 35A40.

\section{INTRODUCTION.}

We have obtained soliton lattice solutions when we specify an integration path for a nonintegrable system of equations [1]. The question we pose is whether there exists some operation that preserves the form of the basic equations, preferably preserving the soliton concept, but rearranging the multiparticles so they have a more disorderly arrangement.

What we have ultimately in mind is as follows. Disorderliness is a feature associated with quantum systems. Although we shall not be able to make any definite statements regarding the basis of quantum mechanics at this time, a first step along the way would be whether we can induce a disorderliness (in a natural way) in a classical system by some mathematical operation that does not affect the form of the basic equations.

It is well known that nonlinear systems can simulate chaotic systems. However, quantum mechanics is present in even the simplest free field situations where the basic equations have the appearance of being very simple. In our approach we will not tinker with the equations themselves, 
but seek some other way to simulate disorderliness. We have chosen to study a lattice system as this is the simplest multiparticle system that we can deal with. Effects that we consider to be of a quantum nature should appear even with such a simple system.

The results of reference [2] suggest that the question posed in the first paragraph has already been answered in the affirmative. However, since the time of reference [2] we have had access to considerably more computer time and so we have been able to further study systems such as the one appearing in Figure 3 of reference [2], over much greater regions and with more accurate grids. With the additional computer time we were able to uncover a rather rigid symmetry in each quadrant of the $x, y$ plane (for data leading to loop soliton lattice systems when we specify an integration path). As an example. note Figure 7 of reference [5]. This is the same system originally studied in Figure 3 of reference [2]. Furthermore, the different soliton loop lattice systems studied in reference [5] all showed the same type symmetry. Consider the maps of reference [5] of the + quadrant which occur when we employ the integration scheme of reference [3]. When we pass to the right of the $x=-y$ line, we find for fixed $y$ that maxima (minima) of the same magnitude are repeated in $x$ for fixed spacings $\Delta x$.

Thus, on the one hand computer runs for regions close to the origin suggest a more disorderly arrangement of planar maxima (minima) as in Figure 3 of reference [2]. On the other hand, with a bigger picture we see a repetitive pattern for planar maxima and minima to the right of the $x=-y$ line' as in reference [5].

In the ++ quadrant the location of planar maxima (minima) is regular as we alter $x$ to the right of the $x=+y$ line. All four quadrants have a $x=+y$ or $x=-y$ type symmetry.

The symmetry in the +- quadrant to the left of the $x=-y$ line occurs when we alter $y$ for fixed $x$.

The $x= \pm y$ lines appear in a basic way in the above symmetry. We note that the integration scheme, [3], [4] in the $x, y$ plane, involves one step in $x$ and one step in $y$ before we sum contributions. Thus, the $x= \pm y$ symmetry is likely to have its roots in the nature of the integration scheme (when combined with a lattice as in reference [5]).

Getting back to the problem posed in the first paragraph. Can we introduce an operation that preserves the basic equations and yet rearranges the lattice particles in what appears to he a disorderly way? In reference [2] we have shown that we can do this in a small region, but we have not succeeded in doing this in the large (reference [5]). We shall thus rephrase the question in paragraph 1. Can we enlarge the nonsymmetric region obtained in reference [2] by some operation that does not alter the form of the basic equations? This will be the goal of the present study.

\section{INTEGRATION IN $Z$.}

We consider date discussed in reference [6], namely:

(i) $\Gamma_{32}^{1}=1.0 \quad \Gamma_{31}^{2}=-1.0$

$\Gamma_{12}^{3}=-1.0 \quad \Gamma_{21}^{3}=1.0$

with the other $\Gamma_{j k}^{i}=0$. To get away from such simple values we integrate along $x$, then $y$, then $z$, then $y$, then $x$, and then $z$ going 700 points for each segment with a grid size of .003 . The resulting $\Gamma_{j k}^{i}$ are then used as origin point data. The system is one of three space-time dimensions.

In Figure 1 we display results using the integration scheme of reference [3] for the component $\Gamma_{33}^{1}$ in the +- quadrant. We recognize the symmetry discussed in Section 1 . The grid used is .009375 . The soliton magnitude is .49 as emphasized in [6]. We then made computer runs to $z=101$ in units of .009375 . This is a coarse grid and this shows up as the soliton magnitude drops to .43 in some instances (see Figure 2). However, the symmetry of regularity of maxima (minima) for fixed $y$ as we increase $x$ to the right of the $x=-y$ line, is still present even though contour lines have changed in a substantial way in this interval.

The symmetry of repeating to the right of the $x=-y$ line appears even if we do not just focus 
on maxima (minima). Consider, as an example, columns of numbers appearing in the map of Figure 1 at $x=9,37$, and 65 (in units of $24 \times .009375$ ) for the component $\Gamma_{33}^{1}$. These are given in Table I. Numbers are truncated after two decimal places.

$\begin{array}{llc}\underline{x=9} & \underline{x=37} & \underline{x=65} \\ .13 & .13 & .13 \\ .20 & .20 & .20 \\ .23 & .23 & .23 \\ .21 & .20 & .20 \\ .12 & .12 & .12\end{array}$

Table I Columns of Numbers Taken from the Map of Figure 1 Showing Repetition of $\Gamma_{33}^{1}$ for $x=9,37,65$, at $z=0$

In Table II we given the corresponding columns at $x=101$ in units of .009375 .

$\begin{array}{ccc}\frac{x=9}{.02} & \frac{x=37}{.02} & \underline{x=65} \\ -.04 & -.04 & .02 \\ -.13 & -.13 & -.04 \\ -.33 & -.33 & -.24 \\ & -.33\end{array}$

Table II Columns of Numbers Showing Repetition of $\Gamma_{33}^{1}$ for $x=9,37,65$ in the +- quadrant for $z=101$

Thus, the symmetry of repeating in $x$ has not been avoided by integrating in $z$ to 101 . The symmetry sets in to the right of the $x=-y$ line and appears when $x$ is increased by 28 units. It is true for $z=0$ and $z=101$ as well as for the $z$ 's studied in between.

We mention steps taken to verify the correctness of the computer program. Close to the origin results were checked to five decimal places for points on an individual basis. The integration scheme of reference [3] was in this way checked against programs used previously. A discussion of checks on the original program is found in reference [1].

As an additional check we tested the integration scheme of reference [3] using data that satisfied the integrability equations. The integration scheme gives the same answers as the conventional calculus in this case and this was checked using previous programs. The parameters used for long runs were the same for the test systems as for the runs appearing in this paper.

In reference [6] we note that the data of this section had the interesting property that trajectories of solitons could not be followed in "time" (the $z$ axis is referred to as the "time" axis) due to solitons appearing and disappearing.

3. EFFECT OF ALTERING THE MAGNITUDES OF ORIGIN POINT DATA.

Consider the following $\Gamma_{j k}^{t}$

(2) $\begin{aligned} \Gamma_{32}^{1}=1.0 & \Gamma_{31}^{2}=-1.0\end{aligned}$

$\Gamma_{23}^{1}=1.0 \quad \Gamma_{13}^{2}=-1.0$

$\Gamma_{21}^{3}=1.0 \quad \Gamma_{12}^{3}=-1.0$

with the other $\Gamma_{j k}^{i}=0$. This intrinsically is a three dimensional system. It gives rise to a soliton lattice when we specify an integration path.

In this section we will study the effect of altering magnitudes of the six $\Gamma_{j k}^{z}$ appearing in (2).

We shall use the data (1) together with the 4-dimensional $e^{\alpha}$, of reference [5] as a comparison system. A map of the data in the +- quadrant for $\Gamma_{1 \mathrm{i}}^{1}$ using the integration scheme of reference [3] and [4] is given in Figure 3. Figure 3 is done with the same grid size and over the same region as Figures $5,7,13$ and thus we can investigate whether our methods enlarge the nonsymmetric 
region. The grid used is .028125 which is a coarse grid but it is accurate enough to establish the symmetry discussed in Section 2.

We next consider the data

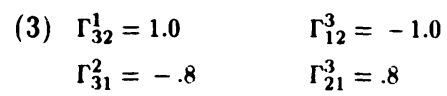

We use the following $e^{\alpha}$,

(4) $e^{\alpha},=\left(\begin{array}{cccc}.88 & -.42 & -.32 & 0.0 \\ .5 & .9 & -.425 & 0.0 \\ .2 & -.55 & 1.0 & 0.0 \\ 0.0 & 0.0 & 0.0 & 1.0\end{array}\right)$

The resulting map is given in Figure 4 when we specify a path in the usual way by integrating over $z$, then $y$, then $x$. The grid is .075 and the spacing between points is .3 . The map is at $z=-.3$. The system is that of a soliton lattice with soliton magnitude $.16 \pm .02$. The solitons remain stationary in time due to the underlying three dimensional character of the system.

In Figure 5 we show a +- quadrant map using the integration scheme of reference [3]. In comparing with Figure 3 we see that the nonsymmetric region has been enlarged. The number of maxima (minima) in the region $y=-3$ to -5 in units of .3 is now 6 before repetition occurs.

We next consider.

(5) $\Gamma_{23}^{1}=.8 \quad \Gamma_{13}^{2}=-.6 \quad \Gamma_{12}^{3}=-.6$

$\Gamma_{32}^{1}=1.0 \quad \Gamma_{31}^{2}=-1.0 \quad \Gamma_{21}^{3}=.8$

with the other $\Gamma_{\beta \gamma}^{\alpha}$ zero. We use the same $e^{\alpha},(4)$, as before. When we specify an integration path we get the map of Figure 6 . In this map $z=-.6$. We again see a soliton lattice, with soliton magnitude $.13 \pm .01$. The +.10 and -.10 contour lines are repeated in an alternating manner as a function of $y$ and as a function of $x$. The \pm .10 contour lines with an uncertainty of \pm .01 have similar structure.

In Figure 7 we use the integration scheme of reference [3]. We see once more that the nonsymmetric region has been enlarged. Further runs indicate that the .12 on the far right on the top of the map is a repeat of the .13 on the left top. There are now eight maxima (minima) within a small $y$ before a repeat occurs. However, the repeat is not quantitative as in Table I and Table II.

In Figure 8 the system is studied with a more accurate .00234375 grid. This map confirms the results of Figure 6 and allows a glimpse of a larger region of space.

Despite the fact that the regularities in $x$ for fixed $y$ to the right of the $x=-y$ line do not appear over a larger region there are still regularities observed. There is an indication that maxima (minima) are evenly spaced on each of a set of lines parallel to the $x=-y$ line. The spacing of maxima (minima) on a line is $20 \pm 5$ units (one unit is $48 \times 2 \times .00234375$ ) although this effect is not seen for all maxima and minima (at least for the grid size used). The contour lines around the repeating maxima (minima) do not have a similar appearance so the impression on looking at Figure 8 is of a largely disorderly pattern at least until repeats in $x$ for fixed $y$ occurs. We see at most 3 maxima (minima) along any line due to limitations incurred by the size of the map.

We have seen that by altering magnitudes of the origin point data we can obtain an enlarged nonsymmetric region. It is not clear if there are limits on such enlargements. It is not feasible to alter magnitudes so that the numbers appearing in the origin point data $\left(\Gamma_{\beta \gamma}^{\alpha}\right.$ and $\left.e^{\alpha}{ }_{i}\right)$ are very large since then we have a problem of numerical errors. For this reason we have restricted magnitudes in (2) to the order of one. We have considered the case of $\Gamma_{32}^{1}=1.5, \Gamma_{13}^{2}=-1.5$ in (5). The resulting map showed that the nonsymmetric region was comparable with Figure 6. 
In both Figure 5 and Figure $\tau$ we see the soliton magnitude as well as maxima (minima) with smaller magnitudes. This is similar to the results of reference [5].

Figures 5 and 7 show that the lattice particles have been rearranged by the integration scheme in a more disorderly way than the lattice for a larger region than that obtained in reference [5] (although some regularities are still seen). This effect arises when magnitudes of the origin point. data are altered.

\section{CONCEPT OF "IMPERFECT" LATTICE.}

Another approach we can take is to find sets of data for which the notion of soliton appears, but in a coarse sense. As an example of this consider the data

(6) $\begin{array}{ll}\Gamma_{23}^{1}=.8 & \Gamma_{20}^{1}=.4 \\ \Gamma_{32}^{1}=1.0 & \Gamma_{02}^{1}=-.5 \\ \Gamma_{13}^{2}=-.6 & \Gamma_{10}^{2}=-.3 \\ \Gamma_{31}^{2}=-1.0 & \Gamma_{01}^{2}=.5 \\ \Gamma_{12}^{3}=-.6 & \Gamma_{12}^{0}=-.3 \\ \Gamma_{21}^{3}=.8 & \Gamma_{21}^{0}=.4\end{array}$

with the other $\Gamma_{\beta \gamma}^{\alpha}$ zero. We use the same four dimensional $e^{\alpha}$, as in reference [5]. When we specify a path we obtain Figure 9.

Figure 9 looks like a lattice. But on closer scrutiny we see, as an example, planar maxima of magnitudes .74 and .80 within the region enclosed by a single .40 contour line. For a perfect lattice these numbers would be the same. Figure 9 was obtained using a .0375 grid which is a finer grid than we generally use in obtaining such lattices. We also see from the computer runs that the .10 contour lines are not as symmetric as the .40 contour lines. Thus, for these reasons, we refer to the system as an "imperfect" lattice. Deviations from a symmetrical situation will also be apparenr when we use the integration scheme of reference [3].

We emphasize that from a numerical point of view we can never say with certainty whether : lattice is perfect or imperfect. We will then rely on visual observations of the maps in s: characterizing a solution.

Maps for different $z$ show that the two maxima (minima) in close proximity eventually merge (both for increasing $z$ and decreasing $z$ ) so that the maxima (minima) lie on a loop. For an imperfect lattice the magnitudes of the maxima (minima) vary somewhat (in this case the range is of the order of ten percent). After the merging of two closely separated planar maxima (minima) there is a marked drop off in the magnitude of the planar maxima (minima). For the system closest to the origin the maximum magnitude drops to .28 at $z=10$ in units of .3 . The magnitudes of the loop was of the order of .80 .

A loop was studied as the time $x^{0}$ evolves in the range $x^{0}=0$ to $x^{0}=8$ in units of .3. We found, in all instances, a magnitude of .80 associated with the loop although as discussed before there was a variation in magnitude with $z$. This result suggests that, in an imperfect sense, we have a soliton loop lattice.

The system was then studied using the integration scheme of reference [3]. The results are given in Figure 10. We mapped a larger region than in Figures 3, 5, and 7 (841 points in $x$ rather than 601 points). We used a grid of .009375 which is smaller than in Figures 3, 5, and 7.

From Figure 10, we do not see the symmetries discussed in Section 1 in the region mapped. Thus, the imperfect lattice is a way to overcome the rigid symmetry seen in reference [5]. However, even though there is no longer a strict symmetry we can recognize that "type" structures do repeat (in a nonexact way). For example, the .70 magnitude surrounded by a .10 contour line 
on the top left is mirrored by a .72 maximum surrounded by a .10 contour line close to the end on the top right. Even though the contour lines have somewhat different shape, therc is a corresponding structure that appears as we increase $x$. There are some eight structures in between such a repeat, similar to the results of Figure 7. In Figure 11, we study the same system with a finer .0046875 grid over a larger region. We can see the repeat of "type" structures more clearly here. We may call this symmetry a correspondence between structures.

As we mentioned before, the magnitude of a maximum (minimum) lies on a loop with a magnitude varying of the order of ten percent in the situation studied when we specify a path. We have looked into the corresponding situation when we use the integration scheme of reference [3]. However, lack of computer time here is a problem as finer grids would be needed. A study of a maximum close to the origin in the + - quadrant shows a similar range of variation for the magnitude of the maxima in the domain studied. The maximum lies on an open string in the interval studied.

Another example of an imperfect lattice is given in Figure 11 of reference [5].

When we take data (5) and use the four dimensional $e^{\alpha}$, of reference [5] we obtain another example of an imperfect lattice. With a .10 contour line close to the origin in the +- quadrant we see a pair of planar maxima of magnitudes .57 and .60 . The effect of using the $e^{\alpha}{ }_{i}$ of reference [5] appears to give results similar to data (6) when use of the integration scheme of reference [3] is made.

\section{HIGHER DIMENSIONS.}

We have seen that the integration scheme of reference [3] can rearrange the lattice particles. In reference [5] we saw a more disorderly system close to the origin, but longer rungs showed a rigid symmetry pattern taking over. We have found in Section 3 that we can enlarge the nonsymmetric region by altering the magnitudes appearing in the origin point data. To what degree such an enlargement is possible is not clear as numerical errors will be a problem if magnitudes become too large. In Section 4, we saw that the symmetry can be overcome if we consider a nonperfect lattice. However, there was a repeat of somewhat similar structures in this case. In this section we shall study an example of a higher dimensional system with an aim of seeing how the situation is affected.

At this point, we do not have reason to favor a particular dimension rather than another We chose to study a complex six dimensional space as we have previously investigated such a sitnation in reference [7].

$x^{1}, x^{2}, x^{3}$ are taken to be real and $x^{4}, x^{5}, x^{6}$ are taken to be pure' imaginary. This then represents a symmetric generalization of the Minkowski hypothesis. Such a system has been studied by other authors as well. For example, note references [8] and [9].

Components of $\Gamma_{j k}^{z}$ are taken to be real if the number of times the indices 4,5 , or 6 appearing in $\Gamma_{j k}^{i}$ is even. $\Gamma_{j k}^{i}$ is pure imaginary if the number of times 4,5 , or 6 appearing in $\Gamma_{j k}{ }_{j k}$ is odd. A similar prescription is taken for $e^{\alpha}$. These reality properties are preserved by the field equations.

Real fields can be said to comprise the subuniverse $U_{1}$, while imaginary fields comprise the subuniverse $U_{2}$. The notion of $U_{1}$ and $U_{2}$ are preserved by the field equations although $U_{1}$ affects $U_{2}$ and vice versa.

We decompose $\Gamma_{\beta \gamma}^{\alpha}$ into real and imaginary parts $\left(A_{\beta \gamma}^{\alpha}, B_{\beta \gamma}^{\alpha}\right.$ are real $)$

(7) $\Gamma_{\beta \gamma}^{\alpha}=A_{\beta \gamma}^{\alpha}+i B_{\beta \gamma}^{\alpha}$

Origin point data is chosen as follows. For nonzero $A_{\beta \gamma}^{\alpha}$ we take 
(8) $A_{23}^{1}=.8 \quad A_{31}^{2}=-1.0$

$$
\begin{array}{ll}
A_{32}^{1}=1.0 & A_{12}^{3}=-.6 \\
A_{13}^{2}=-.6 & A_{21}^{3}=.8
\end{array}
$$

This is the same $\Gamma_{\beta \gamma}^{\alpha}$ used in (5). In addition, our nonzero $B_{\beta \gamma}^{\alpha}$ are taken to be

(9)

$$
\begin{array}{ll}
B_{56}^{4}=.10 & B_{64}^{5}=-.15 \\
B_{65}^{4}=.15 & B_{54}^{6}=.10 \\
B_{46}^{5}=-.13 & B_{45}^{6}=-.13
\end{array}
$$

We also write $\left(f^{\alpha}\right.$, and $g^{\alpha}$, are real $)$

(10) $e^{\alpha}{ }_{i}=f^{\alpha}{ }_{i}+i g^{\alpha}$,

using

(11) $\Gamma_{j k}^{2}=e_{\alpha}{ }^{2} e^{\beta}{ }_{j} e^{\alpha}{ }_{k} \Gamma_{\beta \gamma}^{\alpha}$

We then get the origin point data. $e^{\alpha}$, is the same as appears in reference [7] with the following differences:

$$
\begin{array}{lll}
f_{4}^{5}=.46 & f^{5}{ }_{5}=.88 & f_{6}^{5}=-.35 \\
f_{4}^{6}=.18 & f_{5}^{6}=-.3 & f_{6}^{6}=.94
\end{array}
$$

In Figure 12, we map $A_{11}^{1}$ is the +- quadrant of the $x, y$ plane when we specify a path. The grid used was .15 with every second point printed. We see what appears to be a lattice, but of a different type than we have seen before. Close to the origin we note a .09 contour line. Within this contour we see two planar maxima of magnitude .11. This looks just like what we see for loop lattices (for example, see reference [5]). We study maps with different $z$. We find that the two maxima approach one another in positive as well as negative $z$. The maxima merge at $z=-11$ (in units of .3). Then the magnitude drops off to .085 by $z=-17$. The maxima merge also at $z=2$.

The results thus far have the characteristics of a loop lattice.

We integrated in $x^{0}$ from 0 to 13 (in units of .3 ) with $x^{5}=0$ and $x^{6}=0$. We saw the value of .11 preserved when we studied the region around $z=-13$. The situation is then suggestive of a soliton loop lattice. However, such a simple picture is not realized. We see this when we look at other structures in Figure 12. We note that the magnitudes of the planar maxima (minima) vary from loop to loop. What we see then is a picture consistent with a loop lattice, but with a whole range of different magnitudes.

Table III. Magnitude of Planar Maxima (Minima) at $y=-3 \pm 1$ as

a Function of $x$. The Top Number is Farthest from the Origin in the - - Quadrant. $x$ is Increased as We Go Down the Column So the Bottom Number is Farthest From the Origin in the +- Quadrant. Numbers are Ten Times Actual Numbers.

The spacing between maxima (minima) in the Table is consistent with $\Delta x=24.5 \pm 1(x, y$, and $z$ are all in units of .3).

The results show a repetitive pattern. We see this as follows. The largest planar minima having value $-1.22 \pm .01$ repeats on a regular basis. The smallest magnitude minimum $-.39 \pm .02$, the largest magnitude maximum $1.25 \pm .05$ and the smallest magnitude maximum $.40 \pm .05$ all repeat in a regular manner.

Table III indicates an oscillatory pattern for the magnitude of the planar maxima (minima).

The magnitudes also vary in an oscillatory way as we alter $y$ for fixed $x$. 
The results appear consistent with a loop lattice similar to what we have seen before, but with magnitudes of maxima (minima) varying but in a symmetric oscillatory way. This picture was drawn from results close to the origin and may need to be amended if information further from the origin so dictates.

This type of system was not seen in our previous studies. As the planar maxima (minima) have different magnitudes we have drawn contour lines in Figure 12 having different values associated with them.

In Table III we show how the magnitude of a planar maximum close to the origin varies as a function of $x$ for $y$ fixed at $-3 \pm 1$ (in units of .3). The other coordinates are zero in this table.

\begin{tabular}{|c|c|}
\hline Value of Planar Maximum & Value of Planar Minima \\
\hline 1.12 & -.37 \\
\hline .82 & -.58 \\
\hline .51 & -.88 \\
\hline .35 & -.1 .22 \\
\hline .45 & -1.19 \\
\hline .73 & -1.04 \\
\hline 1.02 & -.71 \\
\hline 1.25 & -.43 \\
\hline 1.15 & -.38 \\
\hline .94 & -.55 \\
\hline .60 & -.84 \\
\hline .40 & -1.13 \\
\hline .44 & -1.23 \\
\hline .67 & -1.06 \\
\hline .97 & -.81 \\
\hline 1.20 & -.49 \\
\hline 1.19 & -.41 \\
\hline .98 & -.52 \\
\hline .68 & -.79 \\
\hline .48 & -1.09 \\
\hline .44 & -1.23 \\
\hline .62 & -1.12 \\
\hline .93 & -.87 \\
\hline 1.19 & -.56 \\
\hline 1.25 & -.40 \\
\hline 1.07 & -.49 \\
\hline .74 & -.77 \\
\hline .47 & -1.05 \\
\hline .42 & -1.23 \\
\hline .58 & -1.17 \\
\hline .86 & -.94 \\
\hline 1.15 & -.63 \\
\hline
\end{tabular}

We next study this system using the integration scheme of reference [3]. The resulting map for $A_{11}^{1}$ is given in Figure 13 in the +- quadrant. The region in the map and the grid size is the same as Figures 3, 5, and 7. We see no evidence for the symmetry discussed in Section 1. 
We then restudied the system with a finer grid of .0046875 over a larger region. The results are given in Figure 14, 15, 16, 17 for the +- quadrant. Figure 15 fits to right of Figure 14. Figure 16 fits to the right of Figure 15 and Figure 17 fits to the right of Figure 16. This confirms the property that the lattice particles are rearranged by the integration scheme without evidence of the rigid symmetry discussed in Section 1, at least in the domain studied.

Although this effect has been obtained in a higher dimensional system we cannot, at this stage, say that the effect occurs as a consequence of the higher dimensions. We have found the rigid symmetry of Section 1 for some other loop lattice systems in both six and eight dimensions. Although we have yet to obtain this effect in four space-time dimensions the possibility exists that a judicious choice of number for the origin point data is responsible for the effect. At any rate, the results of this section show that it is possible to start with a loop lattice, albeit a reasonably complicated one with oscillatory magnitudes of planar maxima (minima), and have the lattice particles rearranged in a way that gives the appearance of a disorderly system (as compared to the lattice) by an integration scheme. The integration scheme arises since the system is nonintegrable. We do not alter the form of the basic nonlinear equations which are taken to be the Aesthetic Field Equations.

Despite the absence of the rigid symmetry described in Section 1, there appear to be other regularities. We see evidence for locations of maxima (minima) along lines parallel to $x=-y$ lines as was observed in Section 3. As with this prior case this is not observed in all instances.

When we specified a path the range of magnitudes for the maxima (minima) was between .12 and .03 (numbers truncated after two places). Using the integration scheme of reference [3] we found the range of the maxima magnitudes to be between .11 and .03 and the minima magnitudes to be between .12 and .03 . Thus, we can say that the range of magnitudes for maxima (minima) which occurs when we specify a path is preserved when we use the integration scheme of reference [3]. The results suggest an oscillatory pattern for the magnitudes although we have not integrated as far in $x$ as we did in Table IV to confirm this.

6. CONCLUSION.

We have studied loop lattice systems when we make use of the integration scheme of reference [3]. Prior to this paper we have seen the lattice particles get rearranged by the integration scheme. Close to the origin the pattern appears disorderly, but in the large a rigid symmetry sets in. In this paper we have shown that by altering the magnitudes of the origin point data we can enlarge the nonsymmetric region. We have shown that certain sets of origin data lead to what we call an "imperfect" lattice when we specify a path. When we apply the integration scheme there is no exact symmetry seen but there is a "correspondence" between structures. For the six dimensional generalized Minkowski system we observe a loop lattice in which magnitudes vary in an oscillatory way when we specify an integration path. When we apply the integration scheme of reference [3] the symmetry pattern is no longer observed in the domain mapped. We cannot say whether this result can be obtained in four dimensions or is a result of the higher dimensions. Although the region for the absence of the rigid symmetry of Section 1 has been enlarged in Section 3 and done away with (in the region mapped) in Section 5, other regularities appear to be still present. 


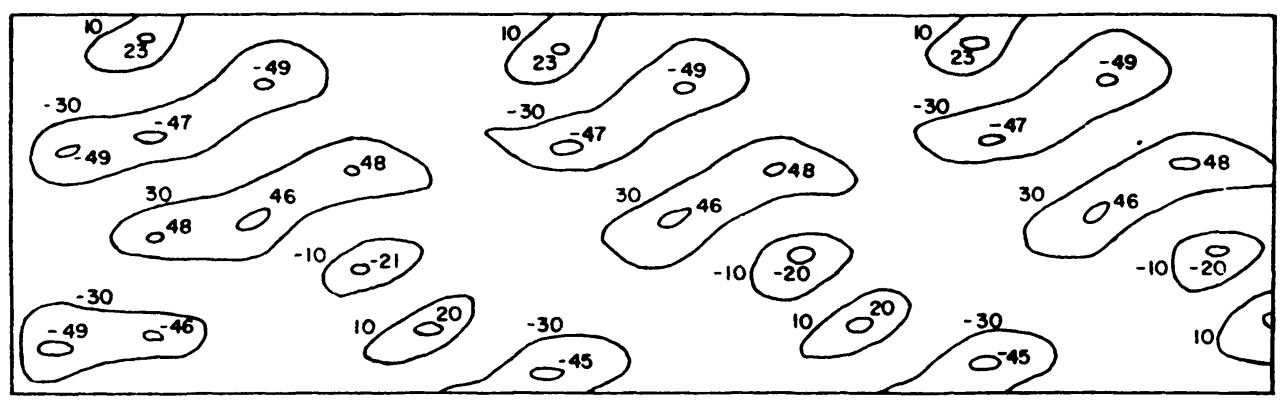

Figure 1. Map of data in Section II at $z=0$ using the integration scheme of [3]. Number of points in map is 2022 by 661 . Grid is .009375 . This and the other maps are in + - quadrant. The numbers in this figure and the other figures are 100 times the actual numbers. Map is for $\Gamma_{33}^{1}$.

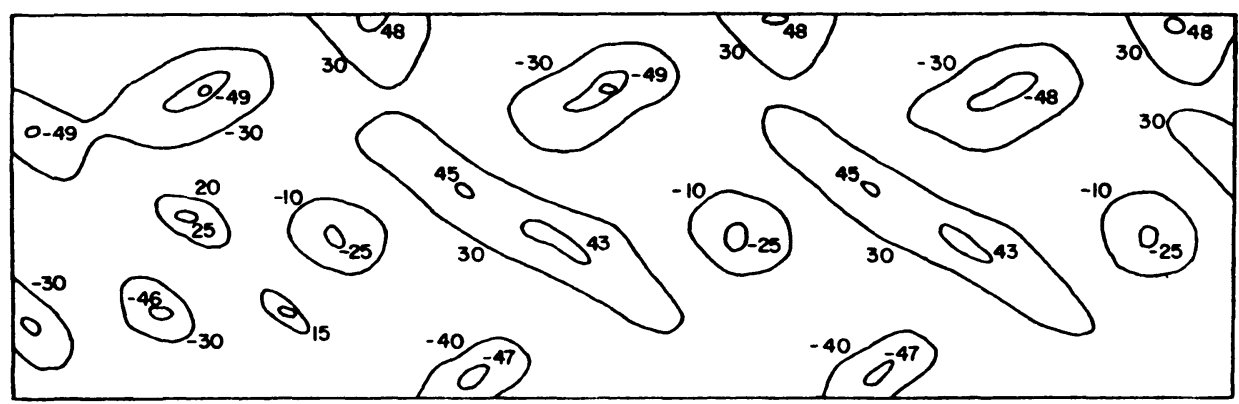

Figure 2. Map of data appearing in Figure 1 at $z=101$ in units of .009375 . The parameters used are the same as in Figure 1. The contour lines have changed significantly from Figure 1 but the symmetry which involves repeating maxima (minima) for fixed $y$ with $x$ being variel, is maintained by the integration scheme. Map is for $\Gamma_{33}^{1}$.

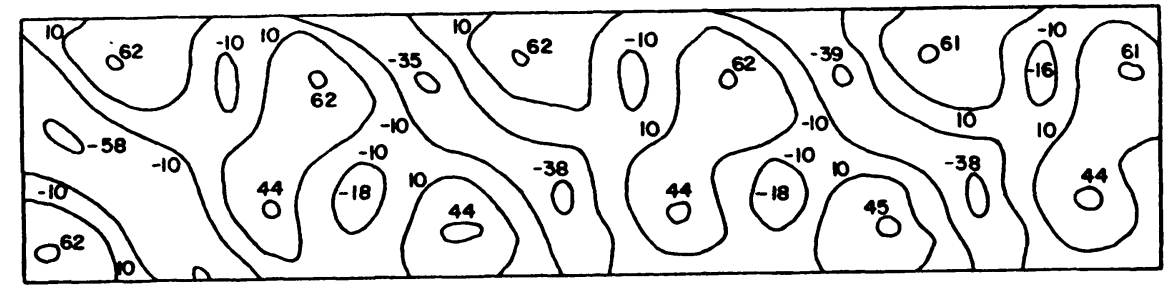

Figure 3. Number of points equals $601 \times 148$. Grid is .028125 . Map is for the data (1) with the 4-dimensional $e^{\alpha}{ }_{i}$ of [5]. Map is for $\Gamma_{11}^{1}$. This map is the same size and with the same parameters as Figures 5, 7, 13 which enables us to compare the size of the nonsymmetric region. 


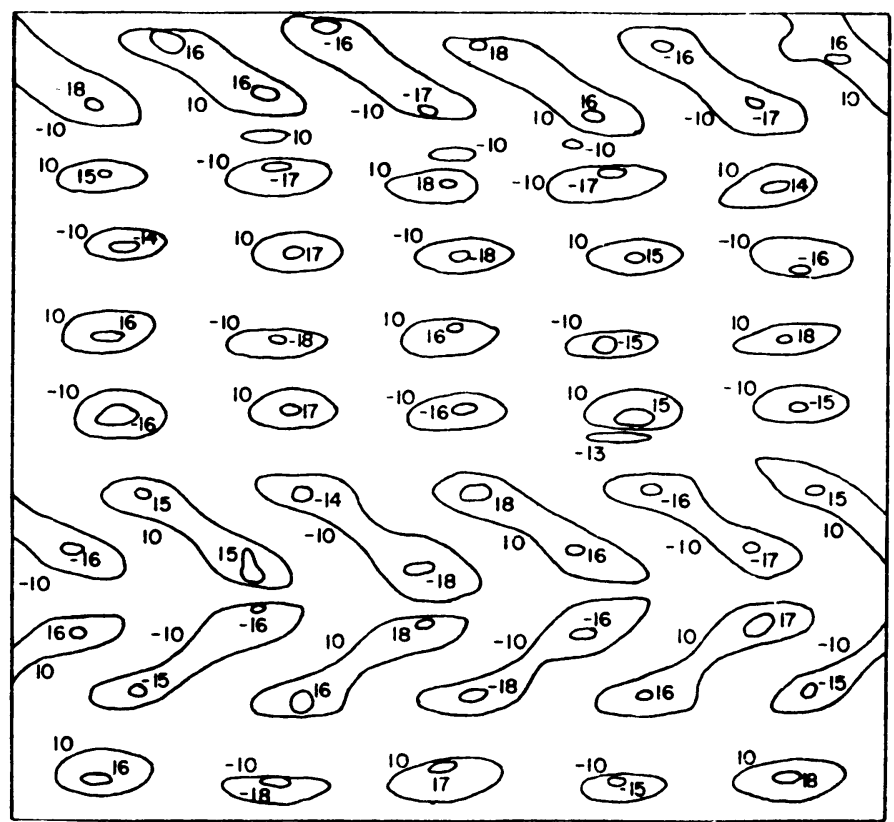

Figure 4. Data based on Equation (3). Map is obtained by specification of path by integrating along $z$, then $y$, then $x$. Grid is .075 . $z$ is equal to -.3 . Results indicate a $.16 \pm .02$ soliton loop lattice that is stationary in time. Map is for $\Gamma_{11}^{1}$.

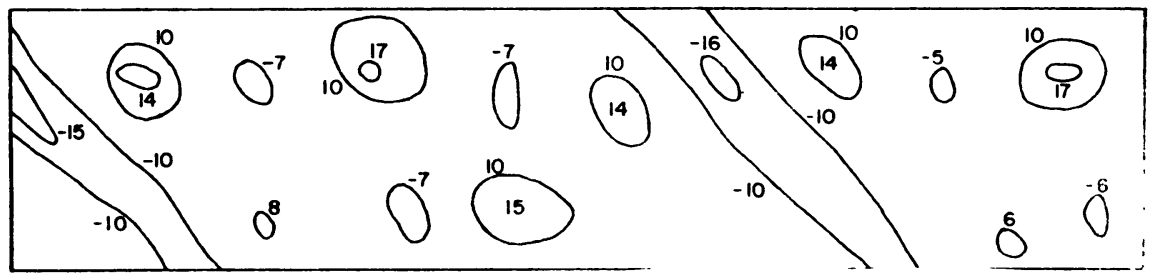

Figure 5. Data based on Equation (3) using the integration scheme of [3]. Comparison with Figure 3 shows that the nonsymmetric region has been enlarged by altering magnitudes of origin point data. Map is for $\Gamma_{11}^{1}$.

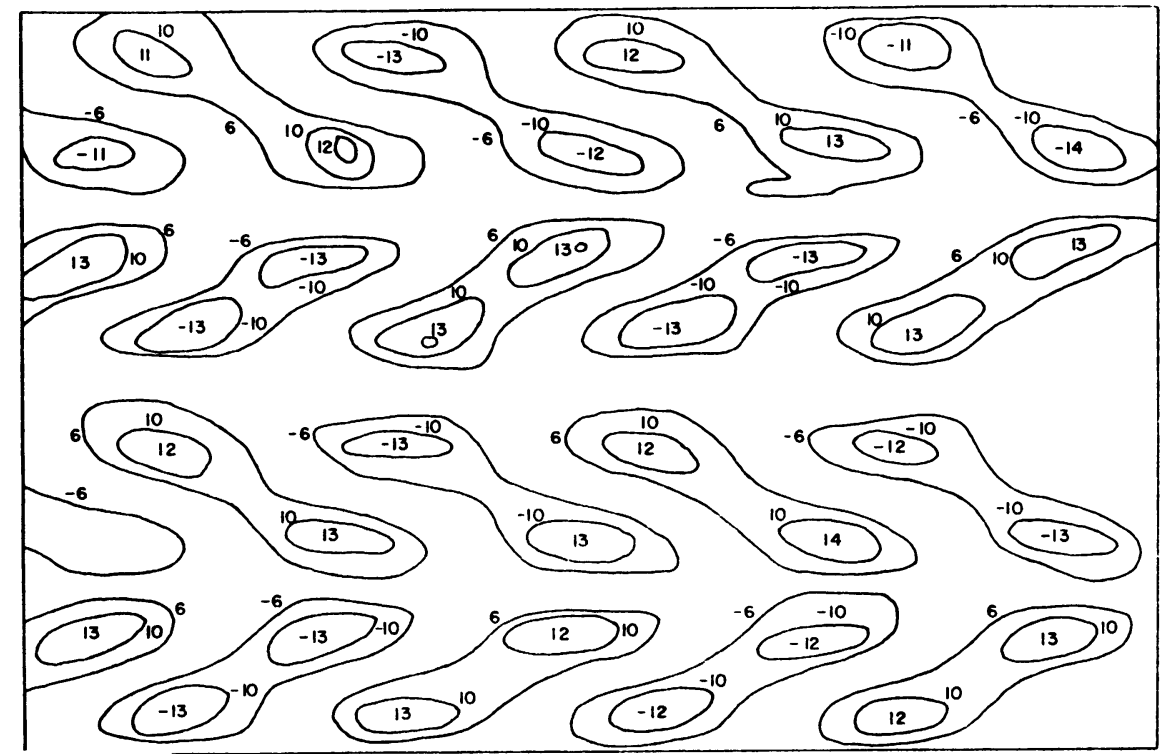

Figure 6. Data based on Equation (5). Map is obtained by specification of path. Grid is .15. Map is for $\Gamma_{11}^{1}$ 


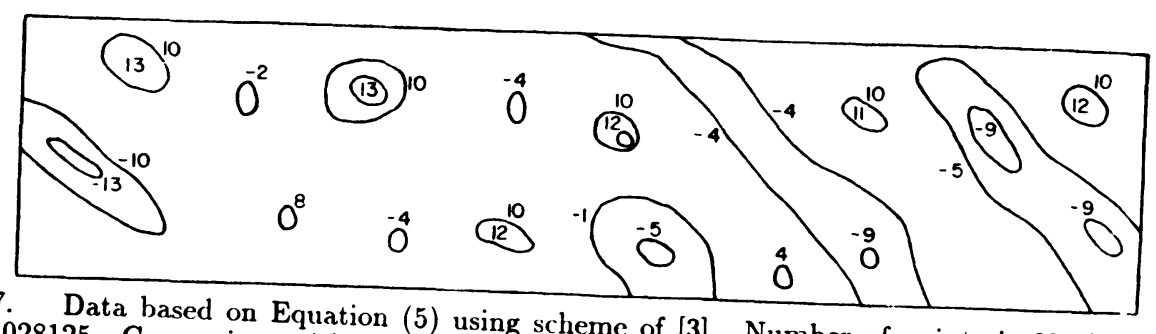

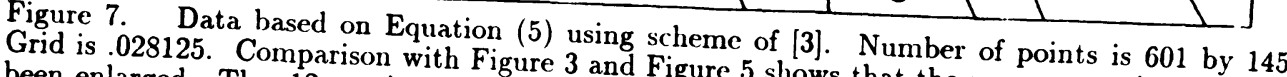
been enlarged. The .12 maximum at the far right is a repeat that the nonsymmetric region has Map is for $\Gamma_{11}^{1}$.

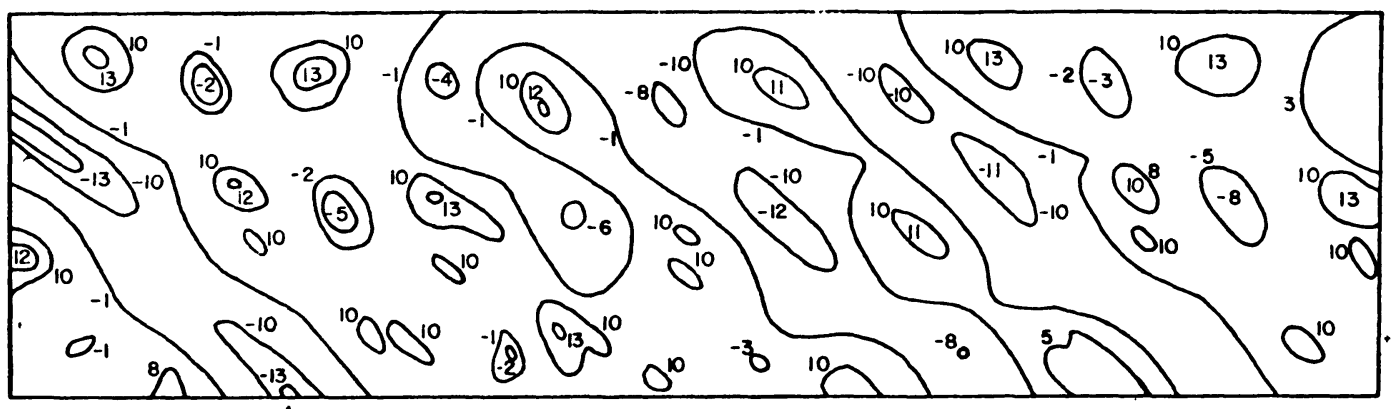

Figure 8. System of Figure 7 is studied with a finer .00234375 grid and a greater region in the + - quadrant is exhibited. Number of points is 14 times 673 by 2689 . This map gives evidence that the .12 on the far right of Figure 7 is a repeat of the .13 maximum at the far left. Map is for $\Gamma_{11}^{1}$.

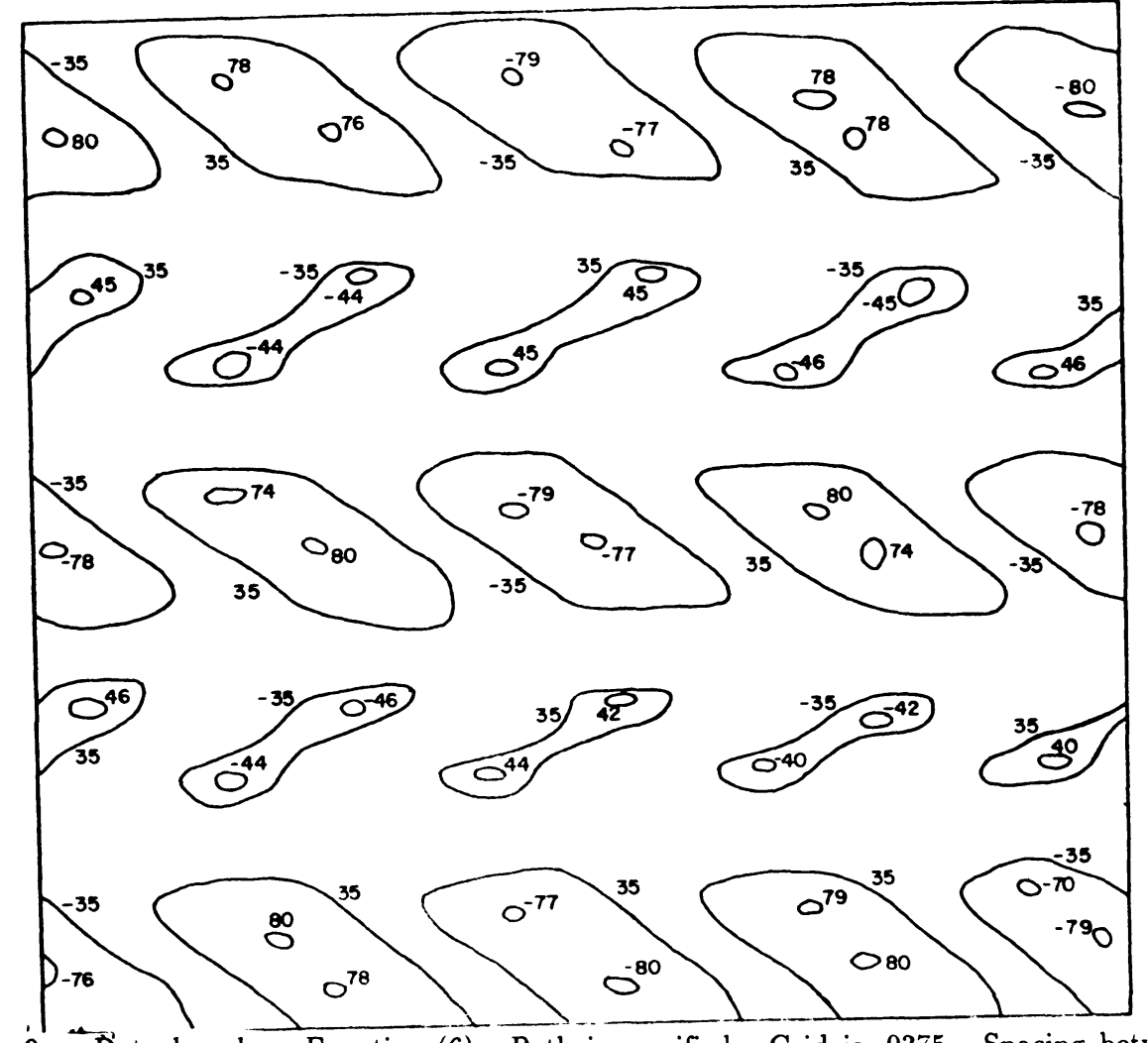

Figure 9. Data based on Equation (6). Path is specified. Grid is .0375. Spacing between points is 8 . Results indicate what we call an "imperfect" lattice. The two maxima (minima). within a contour line have slightly different magnitudes in general. $\Gamma_{11}^{1}$ is mapped. 


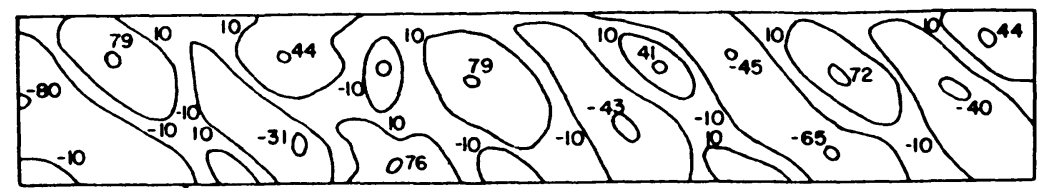

Figure 10. Data based on Equation (6) using integration scheme of reference 3. Number of points is 2521 by 433 . The grid is .009375 . The region mapped is larger than Figures 3, 5, and 7 . No exact symmetry is observed. There is a repeat of similar type structures (for example, the .72 maximum surrounded by the contour line .10 on the right in a coarse sense is a repeat of the .79 maximum surrounded by the .10 contour line appearing on the far left). Map is for $\Gamma_{11}^{1}$.

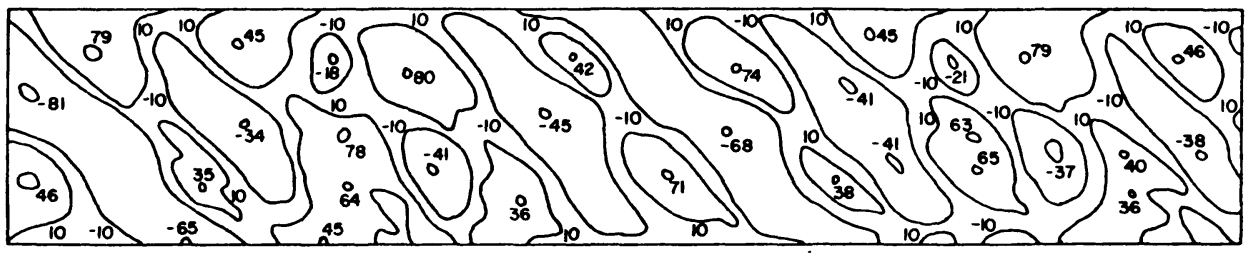

Figure 11. Data based on Equation (6) using a finer .0046875 grid. Number of points is 6 times 1393 by 1393. Map is for $\Gamma_{11}^{1}$.

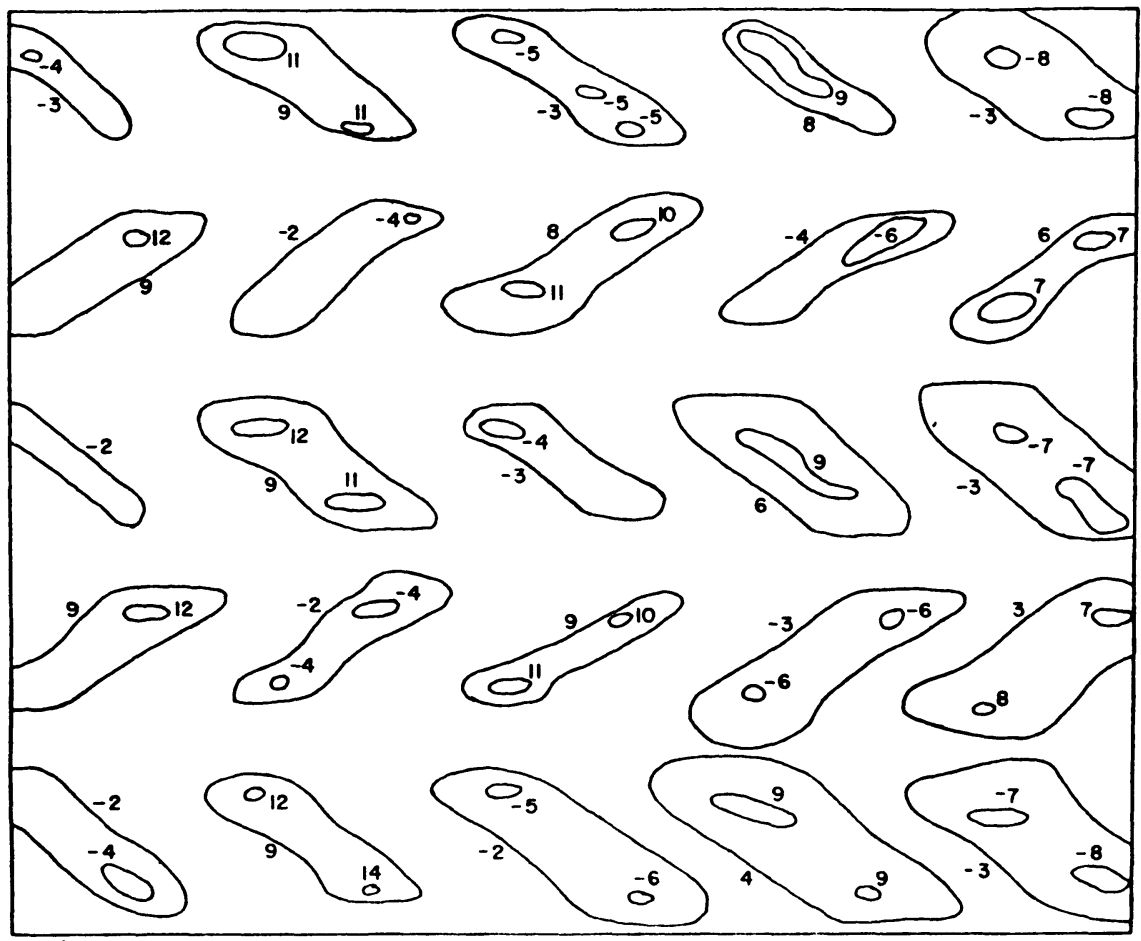

Figure 12. Six-dimensional system of Section V is mapped by specifying an integration path. The value of $z$ is zero. The grid is .15 . This is a loop lattice system in which the magnitudes of the maxima (minima) vary in an oscillatory way. Map is for $A_{11}^{\mathrm{T}}$. 


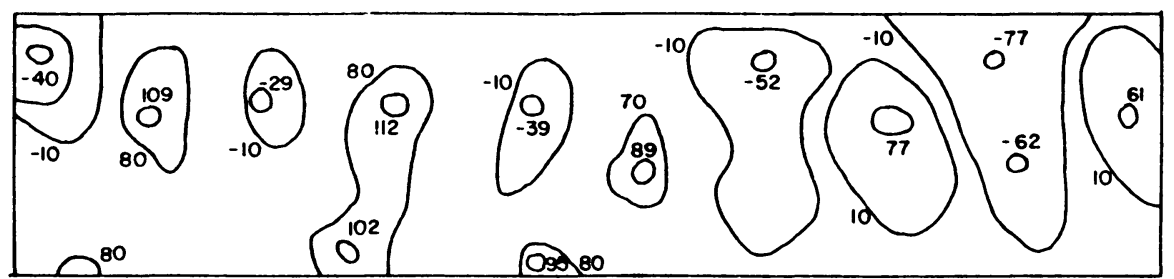

Figure 13. Data of Figure 12 using the integration scheme of [3]. The parameters of the map are the same as Figures 3, 5, and 7. Number of points is 601 by 145 . The grid is .028125 . The numbers are 1000 times the actual numbers. Symmetry of Section I is not observed. Map is for $A_{11}^{1}$.

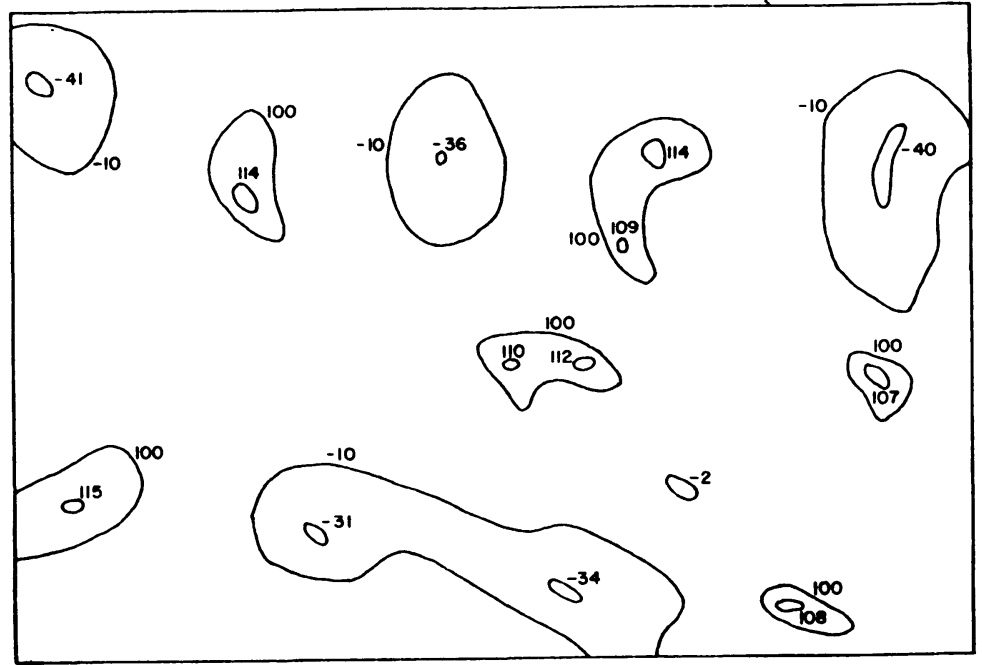

Figure 14. Data of Figure 13 using a finer .0046875 grid over a larger region than Figure 13. Numbers are 1000 times actual numbers. Number of points is 73 plus 20 times 193 hy 1345 points. The symmetries of Section I are not observed in the domain mapped. Map is for $A_{1}^{1}$,
Integration scheme of [3] is used.

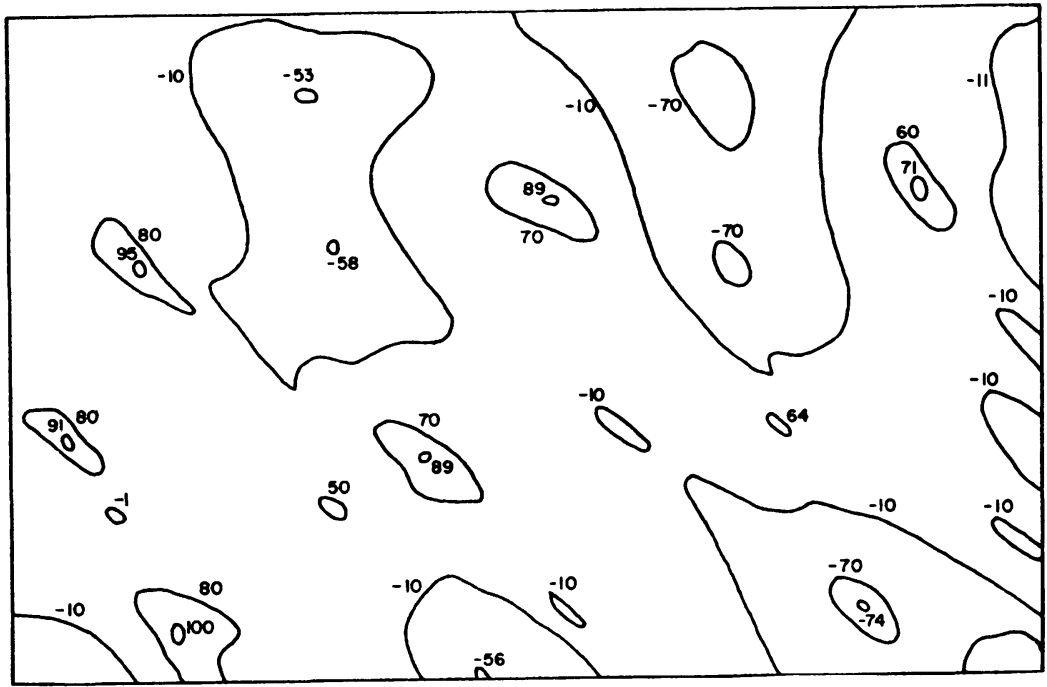

Figure 15. This figure fits to the right of Figure 14. 


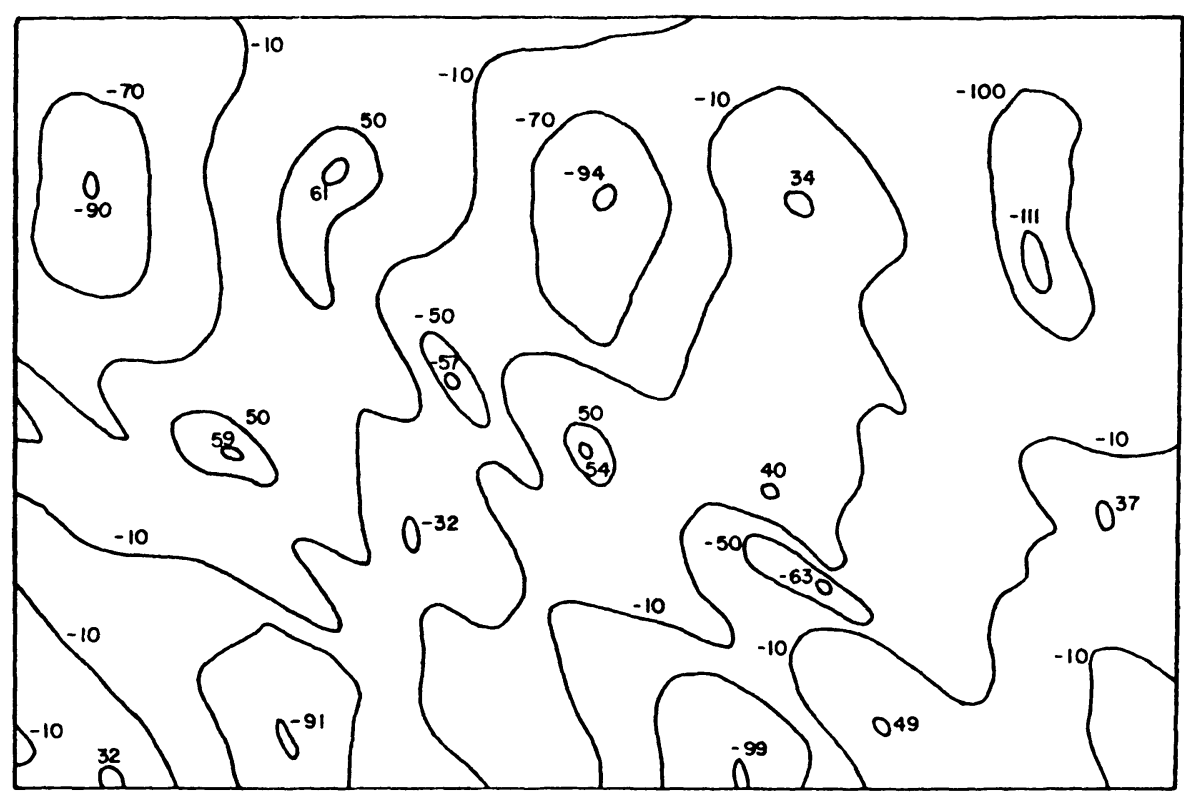

Figure 16. This figure fits to the right of Figure 15.

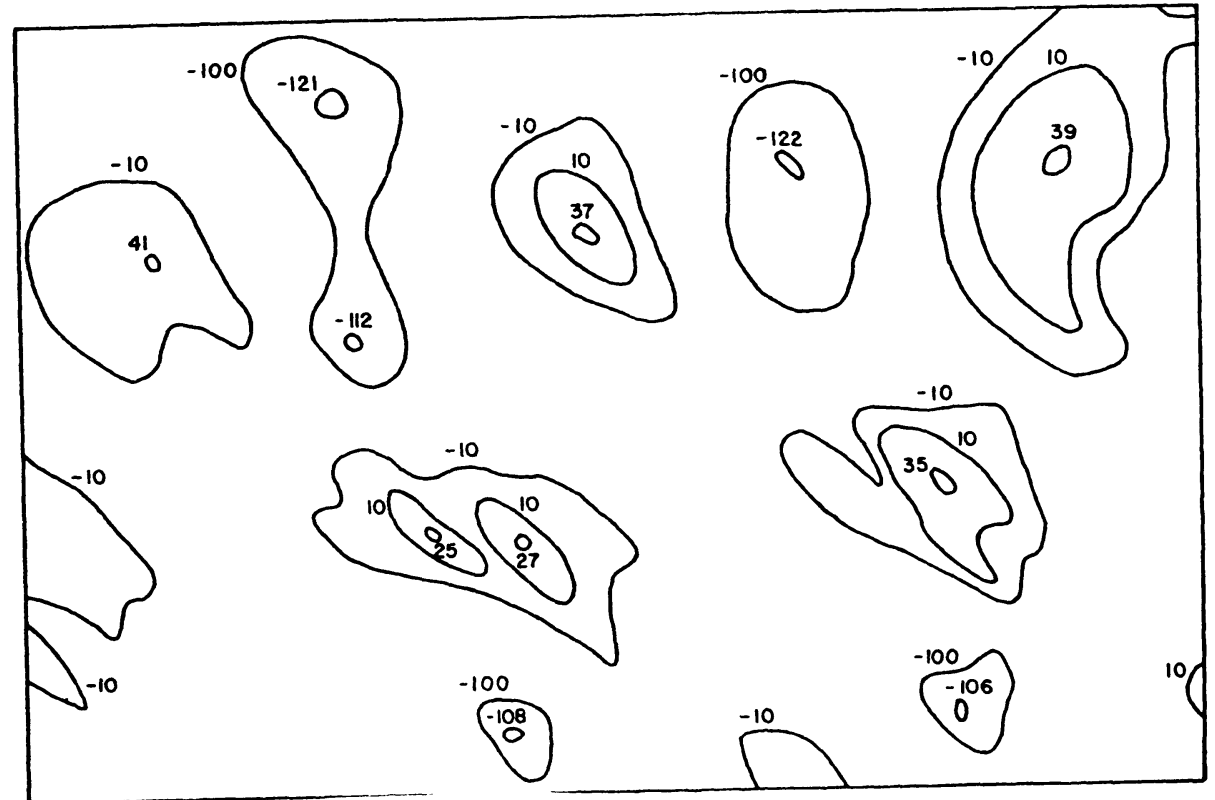

Figure 17. This figure fits to the right of Figure 16. 


\section{REFERENCES}

1. MURASKIN, M. Introduction to derivatives that are not necessary symmetric and a possible role for them in physics, Physics Essays 2 (1989), 375.

2. MURASKIN, M. Three dimensional particle structure using the new approach to nonintegrable aesthetic field theory, Preprint.

3. MURASKIN, M. Alternate approach to no integrability field theory, Mathematical and Computer Modelling 12 (1989), 721.

4. MURASKIN, M. Nonintegrable Systems, Seventh International Conference on Mathematical and Computer Modelling, August 2-5, 1989, Mathematical and Computer Modelling 14 (1990), 64 .

5. MURASKIN, M. Computer two dimensional maps of loop soliton systems using the new approach to no integrability aesthetic field equations, Intern. J. Math. Math. Sci. 15 (1992), 563.

6. MURASKIN, M. Trajectories of lattice particles using the new approach to no integrability, Mathematical and Computer Modelling 12 (1987), 1545.

7. MURASKIN, M. Aesthetic Fields: A two dimensional lattice solution satisfying integrability, Mathematical Modelling 9 (1987), 883.

8. MURAI, Y. On the group of transformation in 6-dimensional space, Prog. Theor. Phys. 11 (1954), 441.

9. PATTY, C. \& SMALLEY, L. Dirac Equation in a Six-Dimensional Spacetime: Temporal Polarization for Subliminal Interactions, Phys. Rev. D32 (1985), 891.

10. COLE, E.A.B. Particle decay in six-dimensional relativity, J. Phys. A. Math. Gen. 13 (1980), 109. 


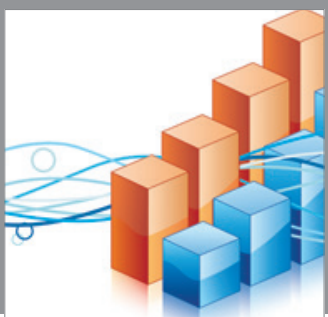

Advances in

Operations Research

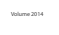

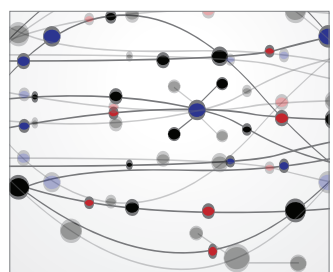

\section{The Scientific} World Journal
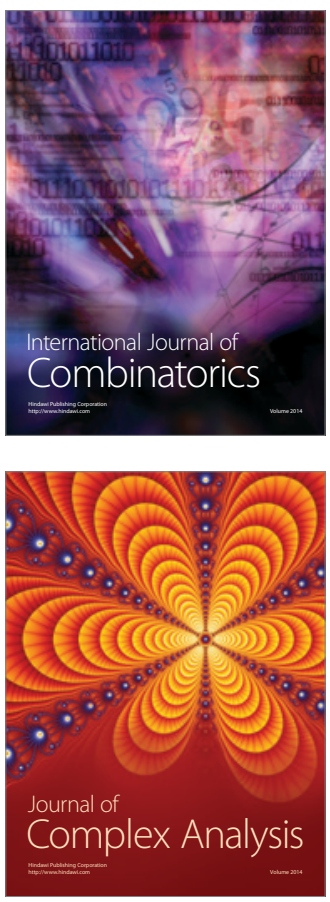

International Journal of

Mathematics and

Mathematical

Sciences
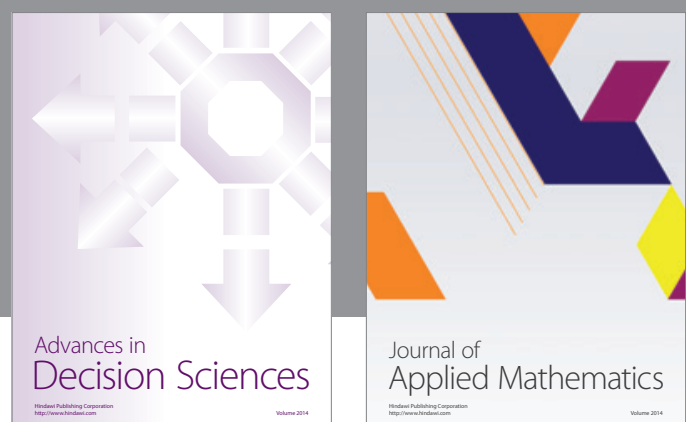

Journal of

Applied Mathematics
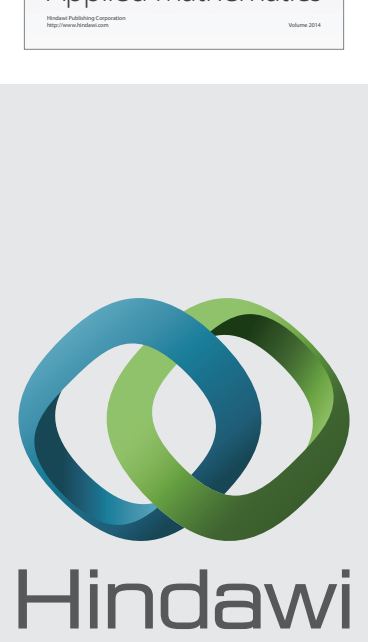

Submit your manuscripts at http://www.hindawi.com
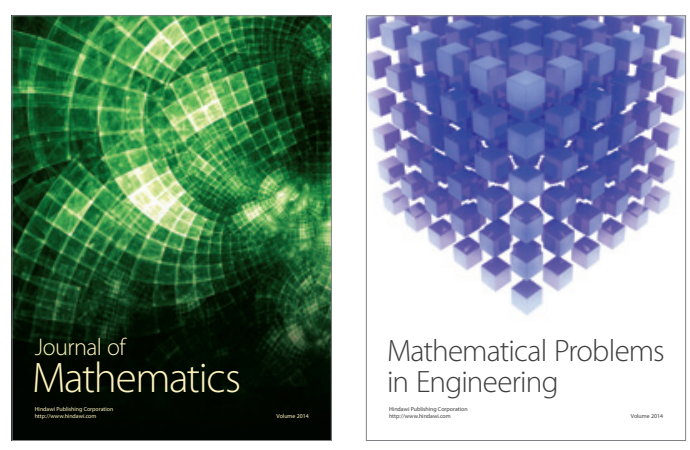

Mathematical Problems in Engineering
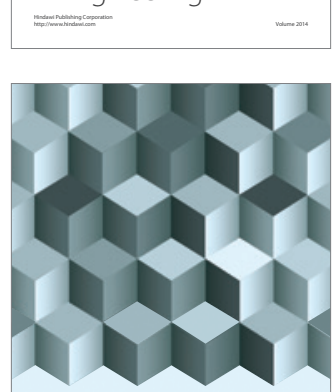

Journal of

Function Spaces
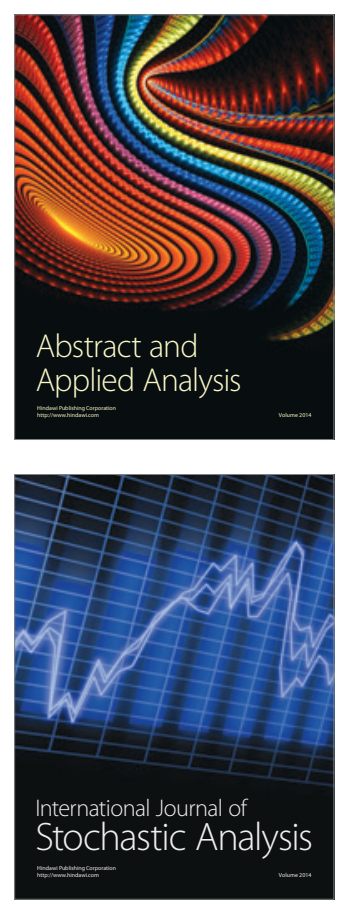

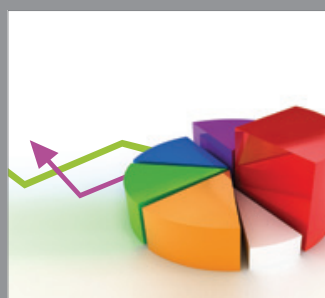

ournal of

Probability and Statistics

Promensencen
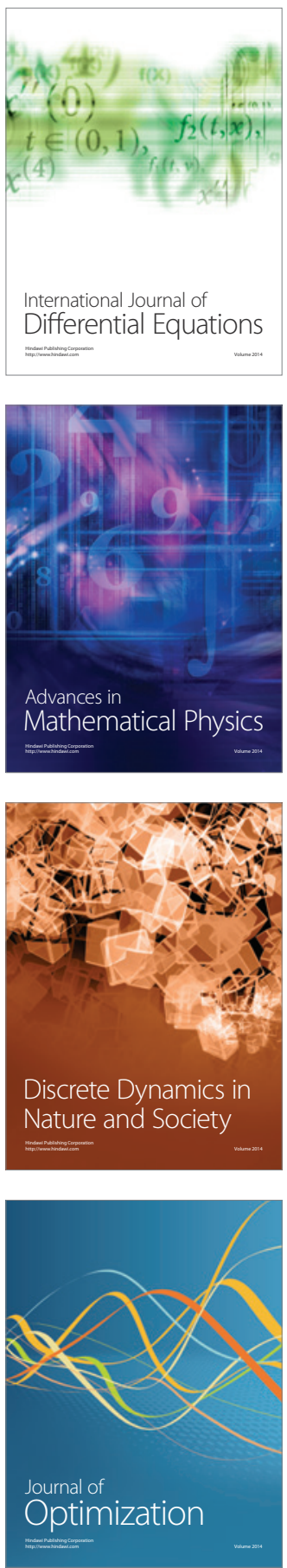